\section{Leave your pet feline good this Christmas}

Looking for a gift for a moggy who has everything, including bad breath? Then you need feline interdental brushes.

The new Cleaned Up Clawed Off range comes in seven different colours and sizes to effectively clean that huge space between your cat's fangs. Wide enough to drive a double decker bus through yet sharp enough to pierce skin, cat's teeth are notorious for collecting food debris, a sure fire way of gum disease developing.

Designed to poke around in your moggy's mouth, Cleaned Up Clawed Off are clinically proven to reduce gum disease in cats by up to $10 \%$, significantly higher than competing brands. They also come in several different flavours, designed to encourage your cat's compliance. Evidence suggests you should begin to clean your cat's teeth as soon as their first fang appears, so the chicken, salmon, tuna, ham and mac ' $n$ ' cheese flavours should bring out the very best in their fangs.

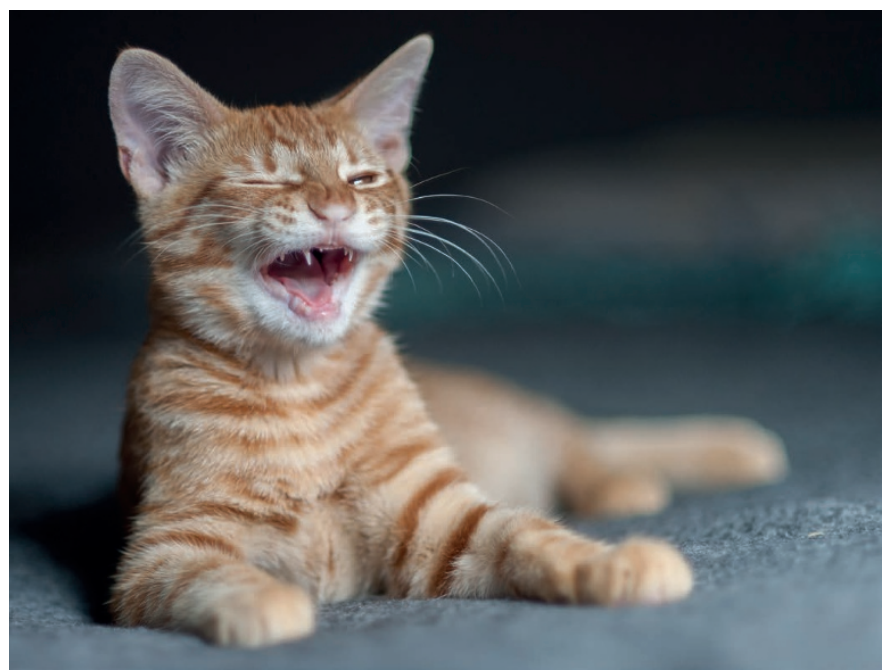

The range also features a line especially for dogs, which comes with an exciting squeaky sound to it.

For further information and to check local stockists visit the Cleaned Up Clawed Off website at www.intermog.com.

\section{A perfect rainbow on your toothbrush}

Brush your way to a brighter future by adding a rainbow to your blue sky thinking. New to the HugeHue range from Custompaste comes Pot of Gold, the unique multi-chromic dentifrice that gives you a perfect rainbow on your toothbrush every time you squeeze.

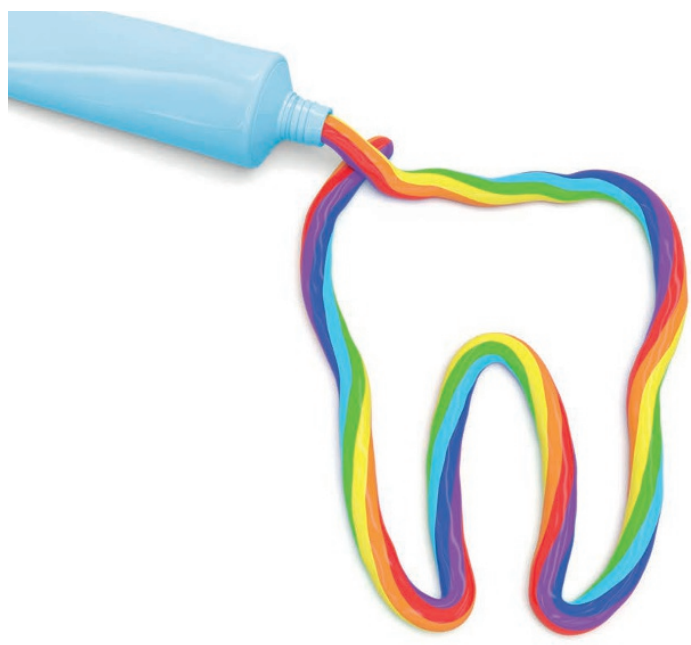

Ideal for encouraging children to brush, Pot of Gold toothpaste has also proven to be a great spirit-lifter for adults as the rainbow landing on the bristles brings a lottery-winning smile even on a grey Monday morning. Innovatively formulated with its ground-breaking gyratory technology, Pot of Gold will produce a perfect rainbow either horizontally for flat head brushes or vertically for smaller, rounded electric toothbrushes. Landing with Red, Orange, Yellow, Green, Blue, Indigo and Violet stripes in perfect sunshine order, Pot of Gold also contains fluoride throughout, melding the colours with prism-like precision to recombine into pure white for the perfect smile.

Pot of Gold joins sister dentifrices Tartan Tooth, available in six different clan tartans including McDonalds and McCloud, and MagnoliaDent suitable for all occasions if colour-matching is an issue when brushing in contrasting bathrooms such as at friends', relatives' or cheap hotels. For further information see www.potofgoldtp.com.

\section{Diamante filling material}

Now you can give your patients the sparkle they always ask for in a filling material. New from SequinDent is Diamante Restorative Solution (DRS) the all in one ompomer-compomer ionomet super composite that twinkles every time your patient smiles, giving them that toothpaste-commercial look they crave.

Ideal for use in both anterior (smile zone) and posterior situations, Diamante Restorative Solution is the all-round cosmetic and functional systemassisted answer to a multitude of conservative dentistry questions. Containing innovate RefractoryRecombitant technology, the diffusion of photon energy across the surface characteristics means that your patients' restorations catch and reflect the maximum available light. Operative in both natural and artificial light DRS bounces

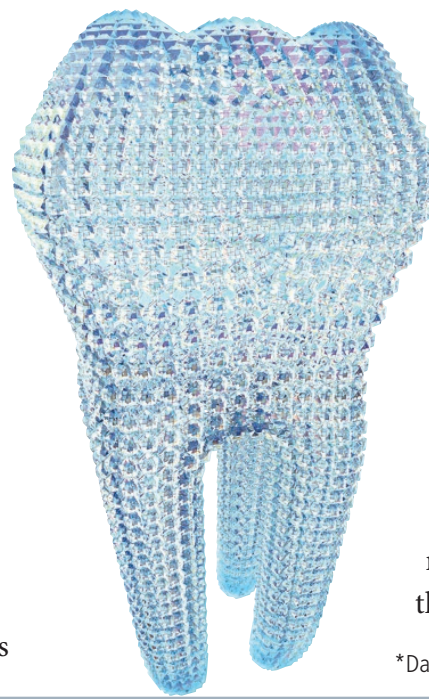

light back out of your patients' mouths for optimum aesthetic appeal and guaranteed conversational impact.

Patients report incredible responses including improved self-confidence, better dating and success in job interviews.*

Also, watch out in 2017 for soon to the market Rhinestone Restorative Solution, the value variant for use within managed healthcare provision providers. For further information, please ask your SequinDent representative or visit Stand A1 at Bling2017 at the NEC; 1 April 2017.

*Data on fileRibusa nis vella vid qui ut quae aut aut quaepud andae. 